\title{
BIOLOGICALLY INSPIRED FILTERS UTILIZING SPECTRAL PROPERTIES OF TOEPLITZ-BLOCK-TOEPLITZ MATRICES
}

\author{
Dragan Vidacic ${ }^{1)}$, Richard A. Messner ${ }^{2)}$ \\ ${ }^{1)}$ IDEXX Laboratories, Inc., Westbrook, ME, 04092, dragan-vidacic@idexx.com \\ ${ }^{2)}$ University of New Hampshire ECE Department, Durham, NH, 03824, rich.messner@unh.edu, www.svpal.unh.edu
}

\begin{abstract}
The construction of filters arising from linear neural networks with feed-backward excitatory-inhibitory connections is presented. Spatially invariant coupling between neurons and the distribution of neuron-receptor units in the form of a uniform square grid yield the TBT (Toeplitz-Block-Toeplitz) connection matrix. Utilizing the relationship between spectral properties of such matrices and their generating functions, the method for construction of recurrent linear networks is addressed. By appropriately bounding the generating function, the connection matrix eigenvalues are kept in the desired range allowing for large matrix inverse to be approximated by a convergent power series. Instead of matrix inversion, the single pass convolution with the filter obtained from the network connection weights is applied when solving the network. For the case of inter-neuron coupling in the form of a function that is expandable in a Fourier series in polar angle, the network response filter is shown to be steerable. Copyright $@$ Research Institute for Intelligent Computer Systems, 2015. All rights reserved.
\end{abstract}

Keywords: Lateral Inhibition Excitation, Recurrent Neural Networks, Toeplitz-Block-Toeplitz Matrices.

\section{INTRODUCTION}

This paper addresses the construction of filters arising from neural networks with feed-backward connections accounting for lateral inhibition and excitation. Such networks are often used when modeling biological vision systems. The well known example is the Hartline-Ratliff equation [1], [2] which describes the mutual inhibitory influences of visual receptor units of the limulus. This discussion is restricted to the simplified, linear model of the limulus equation and its steady state solution as described by:

$$
\overrightarrow{\mathbf{y}}=[\mathbf{I}+\mathbf{B}]^{-1} \overrightarrow{\mathbf{x}}
$$

Vectors $\overrightarrow{\mathbf{x}}$ and $\overrightarrow{\mathbf{y}}$ from (1) are time invariant input and system output respectively while, $\mathbf{B}$ represents the connection matrix. Gutkin and Smith [3] studied the dynamics of linearized recurrent networks in the presence of additive noise and pointed out that the asymptotically attractive steady state in the mean described by (1) is achieved if all eigenvalues of the connection matrix $\mathbf{B}$ have real parts greater than -1 . The linearity between input and output as well as the contrast enhancement effects produced by the 1D network with lateral inhibition characterized by (1) were demonstrated by
Furman [4]. Following Furman's work, the 2D model of such a network was addressed by Messner [5] and later used by Szu and Messner [6] when deriving multiple-channel novelty filters of associative memory. In [5] and [6] it was shown that the network response in the case of a Gaussian inhibitory coupling between neurons can be represented in terms of spatial filtering with bandpass DOG (Difference of Gaussian) kernels providing the analogy with spatial frequency theory of early visual processing [7]. Authors of [8] used the iterative method to solve the network characterized by (1) without identifying the proper network weights that would guarantee convergence of such solution. The linear model of recurrent inhibition with DOG inter-neuron coupling was used to account for a Mach band effect, Herman grid illusion and White's effect [9]. Similar model effectively explained the modified Poggendorff illusion [10]. The network used in [9] yields a simple feed forward filter resulting from the matrix inverse operation. This paper demonstrates how to obtain such filters without explicitly inverting the usually large matrix $[\mathbf{I}+\mathbf{B}]$.

Consider networks with a spatial distribution of sensory units producing the system characterized by the TBT (Toeplitz-Block-Toeplitz) matrix, i.e. the matrix with block Toeplitz structure where each 
block is of Toeplitz type. The network response can be found by using specialized algorithms for such systems. Some of those techniques utilize the persymetry property of the TBT matrix [11], [12] and in the case of large matrices can still be computationally demanding. The solution of a TBT system reformulated as a 2-D deconvolution seen in [13] requires the smoothness of the TBT matrix entries. If the inter-neuron coupling is spatially limited or is extremely small for mutually distant units, the connection matrix becomes block banded with banded blocks and can be solved by techniques presented in [14] and [15]. Alternatively, the convolution with a kernel computed iteratively from the coupling model can be used to obtain a close approximation of the network output [5]. Solving the system by this method can reveal important characteristics of the network impulse response filter. In order to achieve the convergent result, the estimation of spectral properties of TBT matrix becomes critical.

The continuous function, $f:[-\pi, \pi]^{2} \rightarrow R$ generates the TBT matrix $\mathbf{B}_{n, m}$ of structure:

$$
\mathbf{B}_{n, m}=\left[\begin{array}{cccc}
\mathbf{B}_{0} & \mathbf{B}_{1} & \cdots & \mathbf{B}_{n-1} \\
\mathbf{B}_{-1} & \ddots & \ddots & \vdots \\
\vdots & \ddots & \ddots & \mathbf{B}_{1} \\
\mathbf{B}_{-n+1} & \cdots & \mathbf{B}_{-1} & \mathbf{B}_{0}
\end{array}\right]
$$

with Toeplitz blocks:

$$
\mathbf{B}_{l}=\left[\begin{array}{cccc}
b_{l, 0} & b_{l, 1} & \cdots & b_{l, m-1} \\
b_{l,-1} & \ddots & \ddots & \vdots \\
\vdots & \ddots & \ddots & b_{l, 1} \\
b_{l,-m+1} & \cdots & b_{l,-1} & b_{l, 0}
\end{array}\right]
$$

if:

$$
\begin{aligned}
& b_{p, q}=\frac{1}{4 \pi^{2}} \int_{[-\pi, \pi]^{2}} f(x, y) e^{-j(p x+q y)} d x d y \\
& \text { where, } \quad p, q=0, \pm 1, \pm 2, \ldots \ldots
\end{aligned}
$$

The relationship between the spectra of Toeplitz matrix and its generating function was introduced by Grenader and Szego [16]. Following their work, Sierra [17] and Tilli [18] showed that the interval containing the eigenvalues of the TBT matrix is closely related to the properties of the matrix generating function. More precisely, Sierra [17] states that for the function $f$ continuous on the interval $[-\pi, \pi]^{2}$, and not identically constant, for any $n$ and $m$, all the eigenvalues of $\mathbf{B}_{n, m}$ lie in the interval: $\left(\min _{[-\pi, \pi]^{2}} f, \max _{[-\pi, \pi]^{2}} f\right)$

This allows one to characterize the spectral properties of the TBT matrix $\mathbf{B}=\mathbf{B}_{n, m}$ based on the corresponding generating function. Such characterization can be used when choosing neural network inter-neuron coupling functions.

In [5] and [6] the microscopic neuron network solution for the $2 \mathrm{D}$ receptor grid with recurrent lateral inhibition was found by approximating the inverse of the following matrix by a convergent power series:

$$
(\mathbf{I}+\mathbf{B})^{-1}=\mathbf{I}-\mathbf{B}+\mathbf{B}^{2}-\mathbf{B}^{3}+\cdots
$$

Identifying a proper connection matrix $\mathbf{B}$ guaranteeing convergence of this series is essential for valid calculation.

The main contributions of this paper are as follows. Based on conditions for convergence of the matrix power series in (5) and the spectral properties of the TBT matrices, the method for construction of inter-neuron connection models is identified and applied to obtain parameters of five different coupling functions. Once the convergence of (5) is guaranteed, the equivalent filter representing the network impulse response is pre-computed explicitly based solely on the connection model. Finally, it is shown that for the inter-neuron coupling in the form of a function expandable in a Fourier series in polar angle, the network behaves as a steerable [19] filter.

\section{CONSTRUCTION AND SOLUTION OF RECURRENT NEURAL NETWORK}

The simplified form of Hartline-Ratlif equation for the $n \times m$ 2D systems case with solution given by (1) can be written in the form:

$$
\mathbf{y}=\mathbf{x}-\mathbf{B y}
$$

where $\mathbf{x}$ and $\mathbf{y}$ are $n m \times 1$ vectors representing input and output of the system and $\mathbf{B}$ is the $n m \times n m$ connection matrix. Consider the uniform distribution of receptor-neurons in a 2D square grid with the structure as presented in Fig. 1.

Assuming that inter-neuron coupling is spatially invariant for the sensory distribution given in Fig. 1 the connection matrix is of TBT type. Furthermore, if the coupling depends only on distances between receptors, the TBT connection matrix is symmetric with symmetric blocks. Following the notation from (2) and (3), each block $\mathbf{B}_{l}$ of this matrix represents interactions between units in rows $i$ and $k$ so that $l=k-i$. 


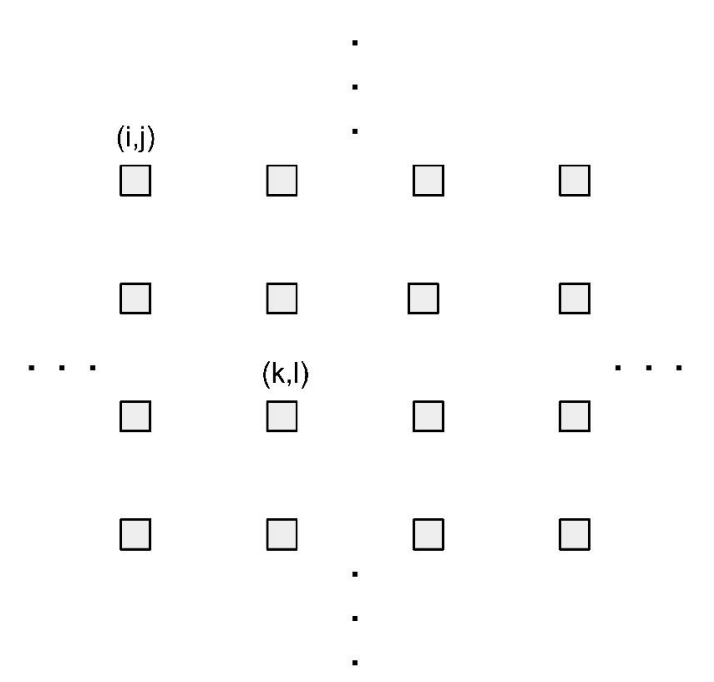

Fig. 1 - Uniform receptor-neuron grid.

The TBT form of matrix $\mathbf{B}$ can efficiently be utilized when solving the system defined by (6). If all the eigenvalues of such a matrix satisfy the condition $\left|\lambda_{i}\right|<1$, the series given in (5) converges [20]. When choosing the inter-neuron coupling model for the receptor grid with recurrent lateral inhibition-excitation, the direct implementation of Sierra's result can ensure adequate spectral properties of TBT connection matrix and allow for the system solution to be found by approximating the matrix inverse in the form of (5).

Instead of using the conventional definition for generating function of TBT matrix, throughout the reminder of this paper it is said that the continuous function $f:[-\pi, \pi]^{2} \rightarrow R$ generates the TBT matrix $\mathbf{B}_{n, m}$ of structure given by (2) and (3) if

$$
b_{p, q}=\frac{1}{4 \pi^{2}} \int_{[-\pi, \pi]^{2}} f^{90^{0}}(x, y) e^{-j(p x+q y)} d x d y
$$

where; $p, q=0, \pm 1, \pm 2, \ldots$

In previous equation, $f^{90^{0}}(x, y)$ represents the rotation of the function $f(x, y)$ by 90 degrees about the origin. The reason for adopting the new definition for the generating function is the fact that the multiplication by the TBT matrix generated according to (7) closely approximates the 2-D convolution with the discrete kernel $b(m, n)^{1}$ comprised of Fourier coefficients of (original, not rotated) function $f$ given as:

${ }^{1}$ Assumption is that this kernel is symmetric.

$$
\begin{aligned}
& b(m, n)=\frac{1}{4 \pi^{2}} \int_{[-\pi, \pi]^{2}} f(x, y) e^{-j(m x+n y)} d x d y \\
& \text { where, } m, n=0, \pm 1, \pm 2, \ldots
\end{aligned}
$$

That is why, for the purposes of the work presented in this paper, it is also said that the function $f(x, y)$ generates the connection function $b(m, n)$ if equation (8) is satisfied.

Proposition 1 (Direct interpretation of Sierra's result for TBT matrices):

If the connection matrix $\mathbf{B}$ from Eq. (6) is generated by the function $f$ that is continuous and not identically constant on $[-\pi, \pi]^{2}$, and if:

$$
\max _{[-\pi, \pi]^{2}}|f(x, y)| \leq 1
$$

the series (5) will converge and the system response can be written in the form:

$$
\mathbf{y}=\left(\mathbf{I}-\mathbf{B}+\mathbf{B}^{2}-\mathbf{B}^{3}+\cdots\right) \mathbf{x}
$$

This proposition defines the method for construction of the network that can be solved without explicit matrix inversion. Note that each matrix-vector product in (10) closely approximates the convolution. Provided convergence is guaranteed, the system response can be realized by the sum of finite number of filters constituting the series (5) [5]. The resulting filter can be precomputed before actual processing takes place. Connection weights from matrix $\mathbf{B}$ as given by (7) can be related to convolution kernel $b(m, n)$ as:

$$
b_{m, n}=b(n,-m)
$$

The impulse response of the network is given as:

$$
h_{t}(m, n)=\delta(m, n)+\sum_{i=1}^{N}(-1)^{n} b^{(i)}(m, n)
$$

where $b^{(i)}(m, n)$ is the discrete connection function convolved with itself $i-1$ times and $\delta(m, n)$ representing the 2D unit impulse. The size of kernel $b(m, n)$ can be quite small if the extent of interneuron coupling is spatially limited. This is in agreement with all practical models addressed herein. As demonstrated in the subsequent text, the choice of generating functions with absolute values of their extremes significantly less than unity improves the speed of convergence and requires fewer filters to be used. 


\subsection{CONSTRUCTION OF CONNECTION MATRIX (PRACTICAL MODELS)}

This section focuses on construction of the network with specific, well defined inter-neuron coupling models. Such models are inspired by functions commonly used to describe receptive fields at various stages of biological vision [21]. The Gaussian kernel as defined can be represented in spatial and frequency domain as follows:

$$
g_{\alpha, \sigma}(x, y)=\alpha e^{-\frac{x^{2}+y^{2}}{2} \sigma^{2}} \stackrel{F}{\longrightarrow} G_{\alpha, \sigma}(u, v)=\frac{2 \pi \alpha}{\sigma^{2}} e^{-\frac{u^{2}+v^{2}}{2 \sigma^{2}}}
$$

Consistent with Eq. (1), (7) and (8), the generating function is denoted as $f(x, y)$ while the connection matrix is labeled as $\mathbf{B}$. It is formed by sampling the connection function $b(u, v)$. From this point on, this correspondence assumes that the generating function is defined on the entire $R^{2}$ domain, i.e. $f: R^{2} \rightarrow R$, but is also narrow-width, with values outside the interval $[-\pi, \pi]^{2}$ close to zero. When this is the case, the weights of the network given by (7) and (8) can be approximated as:

$$
\begin{aligned}
b_{-n, m} & =b(m, n)=\left.b(u, v)\right|_{\substack{u=m U \\
v=n V}} \\
& =\left.\frac{1}{4 \pi^{2}} \int_{(-\infty, \infty)^{2}} f(x, y) e^{-j(u x+v y)} d x d y\right|_{\substack{u=m U \\
v=n V}}
\end{aligned}
$$

where; $U=V=1$, and $m, n=0, \pm 1, \pm 2, \ldots$

Note that while integrating on $[-\pi, \pi]^{2}$ the integral from (14) integrates the same function as that given in (8). In all cases except for the DOG model it is assumed that $\alpha>0$. Since the matrix $\mathbf{B}$ is real, it is necessary that generating functions have real Fourier transform.

\section{Gaussian model}

When dealing with Gaussian coupling, cases with and without self-inhibition are studied. The generating function $f(x, y)$ and the corresponding inter-neuron coupling are given as:

$$
f(x, y)=G_{\alpha, \sigma}(x, y) \stackrel{G e n}{\longrightarrow} b(u, v)=g_{\alpha, \sigma}(u, v)
$$

where operation 'Gen' is defined by Eq. (8). For the network with self-inhibition, the condition for convergence of the series given in (5) becomes:

$$
\frac{2 \pi \alpha}{\sigma^{2}} \leq 1
$$

By eliminating self-inhibition, the elements on the main diagonal of $\mathbf{B}$ are set to zero. In this case the connection matrix spectrum is characterized by:

$$
\lambda_{i} \in\left(-\alpha, \frac{2 \pi \alpha}{\sigma^{2}}-\alpha\right)
$$

Finally, for narrow-width generating functions with $\sigma<\sqrt{\pi}$ the condition for convergence of (5) becomes:

$$
\frac{2 \pi \alpha}{\sigma^{2}}-\alpha \leq 1
$$

\section{DOG model}

For the DOG inter-neuron coupling model, the generating and corresponding connection functions are:

$$
\begin{array}{r}
f(x, y)=G_{\alpha, \sigma}(x, y)-G_{\alpha_{1}, \sigma_{1}}(x, y) \stackrel{\text { Gen }}{\longrightarrow} \\
b(u, v)=g_{\alpha, \sigma}(u, v)-g_{\alpha_{1}, \sigma_{1}}(u, v)
\end{array}
$$

Conditions for the convergence of series given in (5) are set by limiting the extreme values of the generating function or:

$$
\max \left(\left|2 \pi\left(\frac{\alpha}{\sigma^{2}}-\frac{\alpha_{1}}{\sigma_{1}^{2}}\right) \cdot\right| \frac{2 \pi \alpha}{\sigma^{2}} e^{-\frac{\sigma_{1}^{2}}{\sigma_{1}^{2}-\sigma^{2} \ln \frac{\sigma_{1}^{4}}{\sigma_{1} \sigma^{4}}}}-\frac{2 \pi \alpha_{1}}{\sigma_{1}^{2}} e^{-\frac{\sigma^{2}}{\sigma_{1}^{2}-\sigma^{2}} \ln \frac{\alpha \sigma_{1}^{4}}{\sigma_{1} \sigma^{4}}} \mid \leq 1\right.
$$

The Gaussian connection model with unity DC component used in [5] and [6] yields very slow convergence of the series given in (5). If Gaussian or DOG coupling as described by (15) or (19) are used, the network response can still be characterized as the linear combination of Gaussian filters. For example, in the case of Gaussian connection profile without self-inhibition, and assuming a finite number of terms in the series (5) are used, the resulting filter can be rewritten as:

$$
h_{t}=\sum_{i=1}^{N} \mu_{i}\left(g^{(i-1)}-\frac{1}{\xi} g^{(i)}\right)+\mu_{N+1} g^{(N)}
$$

where $g$ is a Gaussian kernel with DC component equal to $\xi$. Coefficients $\mu_{i}$ can easily be obtained from (10) and(12) by replacing the unity matrix with a narrow width Gaussian $g^{(0)}$. Equation (21) 
represents the result similar to that shown by [6] confirming that the network with lateral inhibition behaves as a collection of multiple channel spatial frequency filters.

\section{Difference of Offset Gaussians (DOOG) model}

The generating and the connection functions for the DOOG model used herein are:

$$
\begin{aligned}
& f(x, y) \stackrel{G e n}{\longrightarrow} b(u, v) \\
& f(x, y)=G_{\alpha, \sigma}(x, y)\left[2 \mu \cos \left(x u_{0}+y v_{0}\right)-1\right] \\
& b(u, v)=\mu\left[g_{\alpha, \sigma}\left(u-u_{0}, v-v_{0}\right)+g_{\alpha, \sigma}\left(u+u_{0}, v+v_{0}\right)\right]-g_{\alpha, \sigma}(u, v)
\end{aligned}
$$

By setting $\mu=0.5$, the model similar to the one from [21] consistent with the construction of the DOOG profile based on central differencing of Gaussian function is obtained. In this case:

$$
f(x, y)=-2 G_{\alpha, \sigma}(x, y) \sin ^{2}\left(\frac{x u_{0}+y v_{0}}{2}\right)
$$

Assuming the offset is in the $x$ direction $\left(v_{0}=0\right)$ the series given in (5) converges if:

$f( \pm \sqrt{t}, 0) \geq-1$

where,

$$
t=\frac{\left(\frac{u_{0}}{2 \sigma^{2}}+\frac{u_{0}^{3}}{8}\right)-\sqrt{\left(\frac{u_{0}}{2 \sigma^{2}}+\frac{u_{0}^{3}}{8}\right)^{2}-\frac{u_{0}^{4}}{12 \sigma^{2}}}}{\frac{u_{0}^{3}}{24 \sigma^{2}}}
$$

approximates the location of generating function minimum. The term $t$ of (24) is obtained through the application of a Taylor series expansion.

\section{$2^{\text {nd }}$ Order Derivative of Gaussian (2ODG) model}

The last type of inter-neuron coupling addressed is the Second Order Derivative of Gaussian. Our analysis is based on the derivative in the $x$ direction. Rotation of the generating function by an arbitrary angle causes the rotation of its Fourier transform while the extreme values remain intact yielding the same conditions for convergence of the series given in (5). The 2ODG generating function and its coupling model are defined as:

$$
\begin{aligned}
f(x, y)=-x^{2} G_{\alpha, \sigma}(x, y) \stackrel{\text { Gen }}{\longrightarrow} b(u, v) \\
=\sigma^{2}\left(\sigma^{2} u^{2}-1\right) g_{\alpha, \sigma}(u, v)
\end{aligned}
$$

The condition for convergence of series (5) is:

$$
\frac{4 \pi \alpha}{e} \leq 1
$$

Fig. 2.a. and Fig. 2.b. depict examples of generating functions and the corresponding coupling models that were used.
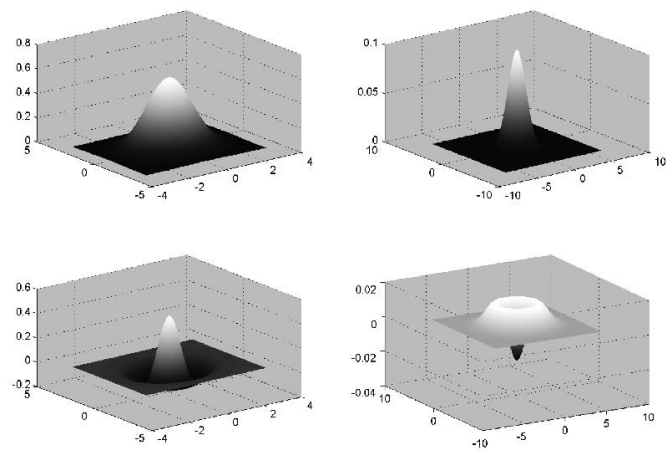

Fig. 2.a - Generating functions (left) and corresponding connection functions (right); Gaussian model (top), DOG model (bottom);
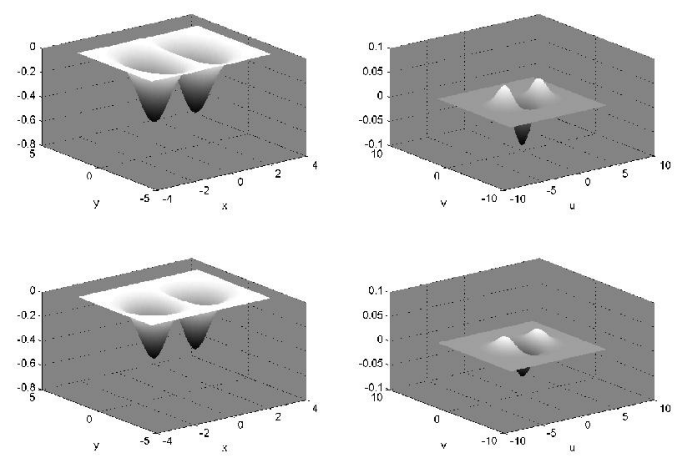

Fig. 2.b - Generating functions (left) and corresponding connection functions (right); DOOG model (top), 2ODG model (bottom).

\subsection{STEERABILITY OF THE RECURRENT NETWORK WITH LATERAL INHIBITION- EXCITATION}

The network filter given in (12) represents the sum of $N+1$ components with each function $b^{(i)}(n, m)$ constructed as $i$ cascades of kernel $b(n, m)$. Consider the connection function $b(x, y)$ (note the replacement of variables $u, v$ with $x, y$ ) that can be represented in polar coordinates $r=\sqrt{x^{2}+y^{2}}$ and $\phi=\arg (x, y)$ as a Fourier series in polar angle: 


$$
b(r, \rho)=\sum_{n=-N}^{N} a_{n}(r) e^{j n \varphi}
$$

The Fourier transform of such a kernel is a function that can also be expanded in a similar way:

$$
B(R, \theta)=\sum_{n=-N}^{N} A_{n}(R) e^{j n \theta}
$$

where,

$$
\begin{aligned}
& R=\sqrt{u^{2}+v^{2}}, \quad \theta=\arg (u, v) \quad \text { and } \\
& A_{n}(R)=2 \pi(-j)^{n} \int_{0}^{\infty} a_{n}(r) J_{n}(2 \pi R r) r d r
\end{aligned}
$$

$J_{n}$ being the Bessel function [22]. Cascading filters represented in frequency domain by (28) produces steerable filter, i.e. arbitrarily rotated function can be synthesized by using the linear combination of basis kernels (for details see [19] and [23]). The steering property holds also for the entire filter given in (12) since the overall sum remains expandable in a Fourier decomposition in polar angle.

It is well known that a 2ODG kernel can be steered by using the linear combination of three basis kernels [19]. As a special case of a kernel given in (27), consider the order $n$ directional derivative of a function $b_{c}(x, y)$ that is circularly symmetric in spatial and frequency domains. The $m-1$ successive convolution of this kernel with itself yields:

$$
\begin{aligned}
& b_{c}^{n, m}(x, y)= \\
& \underbrace{\frac{\partial^{n} b_{c}(x, y)}{\partial x^{n}} * \frac{\partial^{n} b_{c}(x, y)}{\partial x^{n}} * \ldots * \frac{\partial^{n} b_{c}(x, y)}{\partial x^{n}}}_{m-1 \text { convolutions }} \underset{ }{\stackrel{F}{\longrightarrow}(j u)^{n m} B_{C}{ }^{m}(u, v)}
\end{aligned}
$$

The basis for steering of this cascade consists of $n m+1$ functions since the pure real (or imaginary) frequency response is the product of polynomial in $u$ of order $n m$ containing only odd or even terms and circularly symmetric function. The sum of filters:

$$
\begin{aligned}
& h(x, y)= \\
& \sum_{i=1}^{N}(-1)^{i} \underbrace{\frac{\partial^{n} b_{c}(x, y)}{\partial x^{n}} * \frac{\partial^{n} b_{c}(x, y)}{\partial x^{n}} * \ldots * \frac{\partial^{n} b_{c}(x, y)}{\partial x^{n}}}_{i-1 \text { convolutions }}
\end{aligned}
$$

can similarly be represented as a product of polynomial in $x$ of order $n N$ and another circularly symmetric function where the polynomial coefficients may depend on the radius $r$. In this, more general case $2 n N+1$ basis functions are sufficient to steer the function $h(x, y)$. When $b_{c}^{n, m}(x, y)$ is a connection function the overall network impulse response given as:

$$
h_{t}(x, y)=\delta(x, y)+h(x, y)
$$

is steerable as well and represents continuous equivalent of (12). For $n$ even, the number of basis functions that steer (31) is reduced to $n N+1$. For example, in the case of 2 ODG coupling, $2 N+1$ filters steer the network. Steerability can efficiently be used to determine the response of the network for arbitrary orientation of the connection function without performing its actual rotation. Limiting the basis set to a small number of functions by enabling fast convergence of (5) is essential for practical implementations. Fig. 3 depicts components of the steerable impulse response of a network with 2ODG coupling model assuming $N=3$ in continuum; $h_{t}^{\theta}(x, y)$ is an arbitrarily rotated filter while $k_{i}(\theta)$ and $h_{t}^{\theta_{i}}(x, y)$ are the interpolating and basis functions respectively

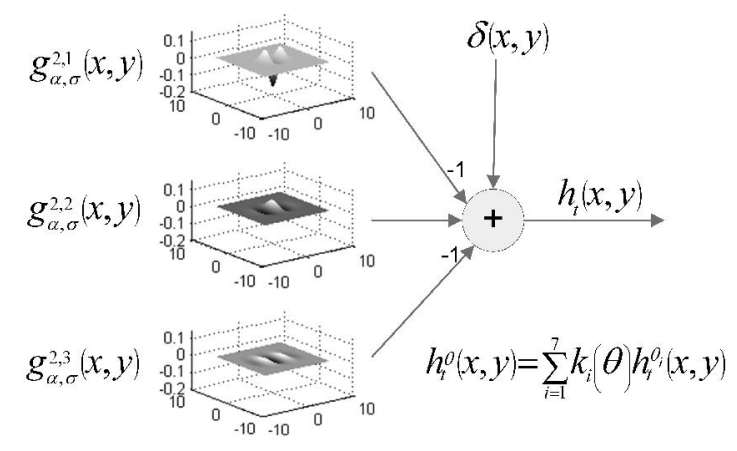

Fig. 3 - Steerable impulse response of the network with $20 D G$ coupling model.

\section{SIMULATION RESULTS}

This section presents simulations obtained when processing synthetic images with networks whose connection weights are constructed using the previously addressed models of inter-neuron coupling. By bounding the generating function, the spectral radius of the connection matrix is maintained in the interval $(0,1)$. This guarantees convergence of the power series given in (5) but it also enables finding of the network solution via the iterative scheme: 


$$
\mathbf{y}_{n+1}=\mathbf{x}-\mathbf{B y}_{n}
$$

previously used in slightly modified form in [8]. Simple algebra proves that this procedure is the equivalent of equation (10). In order to validate the network construction and solution introduced via Proposition 1 and equation (10), the solution obtained by direct convolution with a pre-computed kernel of relatively small size is compared with the solution obtained by application of the iterative scheme given by (32). When solving the system with the iterative method the spatial extent of coupling between neurons was not limited while when using convolution with the pre-computed kernel, the coupling between distant neurons is set to zero. The estimated number of filters $N$ from (12) necessary to produce the convergent result is reached once the energy of the term $b^{(N)}(n, m)$ is extremely small $(0.0002 \%)$ when compared to the energy of the filter $h_{t}(n, m)$. The iterative procedure given by equation (32) is terminated when the average absolute difference between successive solutions is less than a predefined small threshold (for the grayscale images with levels of gray between 0 and 255 this threshold was set to 0.5 ).

When changing parameters of the connection model(s), extremes of generating functions are varied which in turn influences the rate of convergence of the series given in (5). The resulting sufficient number of filters $N$ for all coupling models for each case are shown in Fig. 4 with parameters chosen: Gaussian $\operatorname{model} \sigma=\pi / 3$, DOOG model $\sigma=\pi / 3, u_{0}=2$, 2DOG model $\sigma=\pi / 5, \quad$ DOG $\quad$ model $\quad \alpha_{1}=-0.11$ $\sigma=\pi / 4, \sigma_{1}=\pi / 5$.

Based on Fig. 4, appropriately chosen parameters of the connection function allow the use of a reasonably small $N$. Networks with circular symmetric inter-neuron coupling models (e.g. Gaussian and DOG) produce similar results since the response is not orientation sensitive. Similarly, when non-isotropic models (e.g. DOOG and 2ODG) are used the response is orientation sensitive. Fig. 5 depicts the result obtained by processing a square test pattern of uniform intensity placed on a dark background when using DOG connection model. In this figure a one pass convolution with precomputed kernel using connection model DOG with parameters $\alpha_{1}, \sigma$ and $\sigma_{1}$ identical to those from Fig. 4. and $\alpha=-0.13$ ). The filtered square pattern is shown top and the mid-line resulting profiles for iterative procedure and single-pass convolution is shown bottom. The pre-computed kernel was constructed by using $\mathrm{N}=7$.
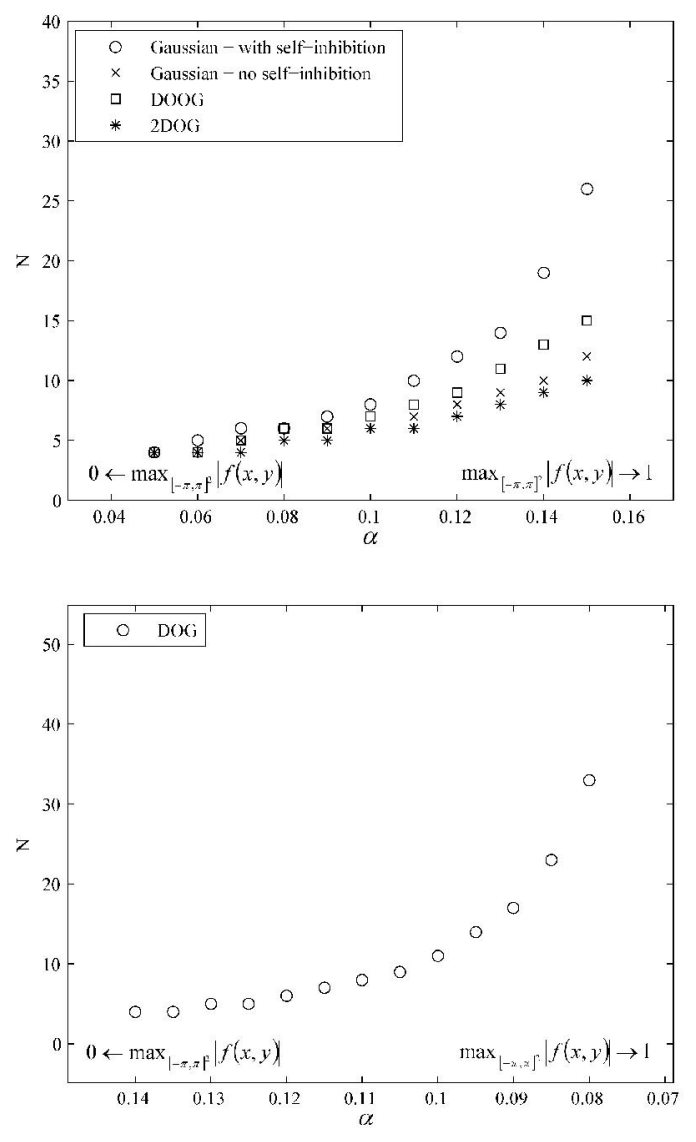

Fig. 4 - The number of sufficient filters versus $\alpha$
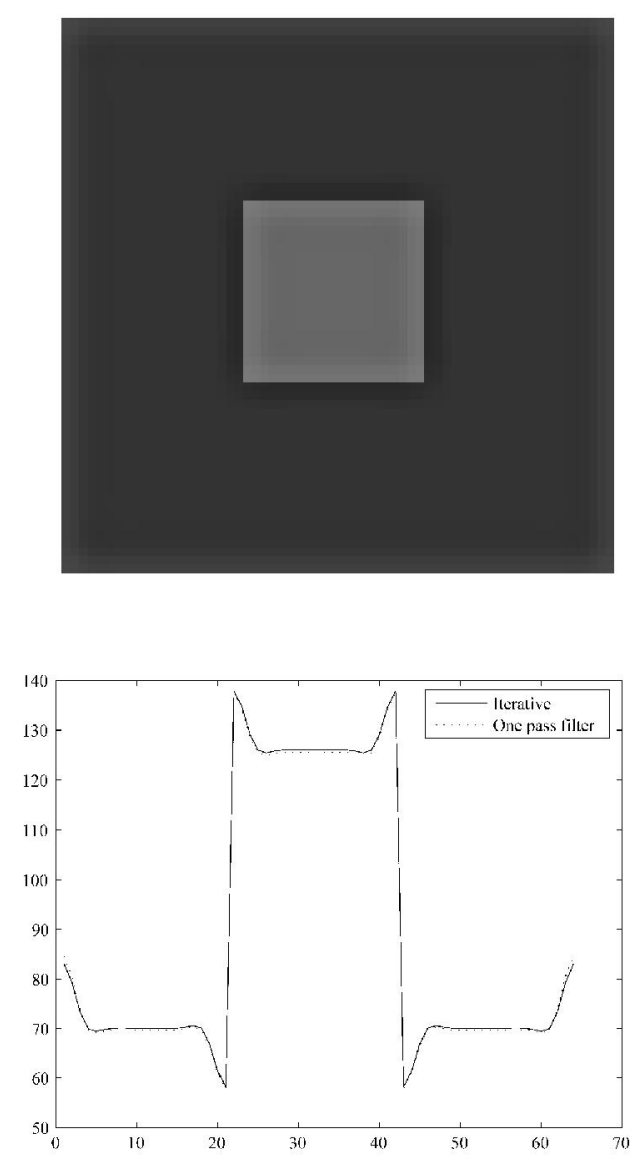

Fig. 5 - One pass convolution with pre-computed kernel. 
As an example for the orientation sensitive model, processing by using a steerable filter constructed from the 2ODG connection function is demonstrated. The number of sufficient filters is chosen based on results described in Fig. 4. Interpolating coefficients necessary for steering are obtained from [19], by using basis functions evenly spaced between 0 and $\pi$. These coefficients are provided in the Table 1. The mesh plots of the network response $h_{t}(m, n)$ and its portion $h(m, n)$ are shown in Fig. 6 for the 2ODG connection model with parameters; $\alpha=0.12, \sigma=\pi / 5, \quad N=7$. Shown are $h(m, n)$ portion top and $h_{t}(m, n)$ bottom.
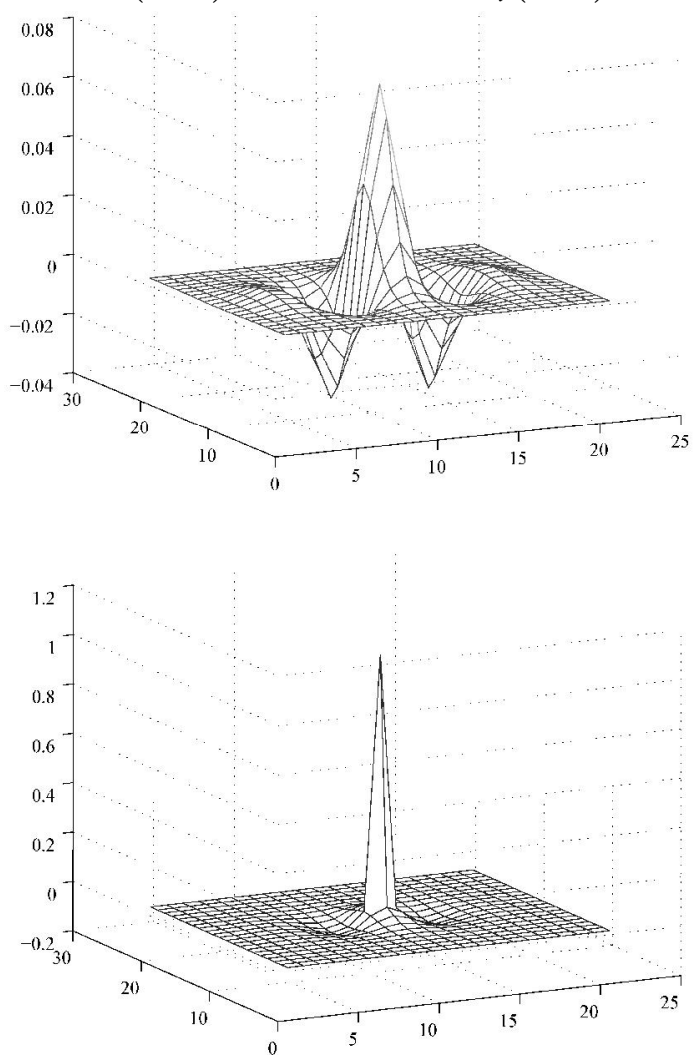

Fig. 6 - Steerable network impulse response.

Table 1. Steering coefficients for the network with 2ODG connection modem $(\alpha=0.12, \sigma=\pi / 5, N=7)$.

\begin{tabular}{|c|c|c|c|}
\hline$i$ & $k_{i}(\pi / 6)$ & $k_{i}(\pi / 2)$ & $k_{i}(5 \pi / 6)$ \\
\hline 1 & 0.1333 & -0.0667 & 0.1333 \\
\hline 2 & -0.2157 & 0.0682 & -0.0996 \\
\hline 3 & 0.6378 & -0.073 & 0.0824 \\
\hline 4 & 0.6378 & 0.0824 & -0.073 \\
\hline 5 & -0.2157 & -0.0996 & 0.0682 \\
\hline 6 & 0.1333 & 0.1333 & -0.0667 \\
\hline 7 & -0.0996 & -0.2157 & 0.0682 \\
\hline 8 & 0.0824 & 0.6378 & -0.073 \\
\hline 9 & -0.073 & 0.6378 & 0.0824 \\
\hline 10 & 0.0682 & -0.2157 & -0.0996 \\
\hline 11 & -0.0667 & 0.1333 & 0.1333 \\
\hline 12 & 0.0682 & -0.0996 & -0.2157 \\
\hline 13 & -0.073 & 0.0824 & 0.6378 \\
\hline 14 & 0.0824 & -0.073 & 0.6378 \\
\hline 15 & -0.0996 & 0.0682 & -0.2157 \\
\hline
\end{tabular}

The results of processing the hexagonal test patterns by the steering the filter $h(m, n)$ are shown in Fig. 7. In this figure steering the network is done with the 2ODG connection model with parameters; $\alpha=0.12, \sigma=\pi / 5$, and $N=7$. A linear combination of 15 filters are used to steer the response to three different orientations
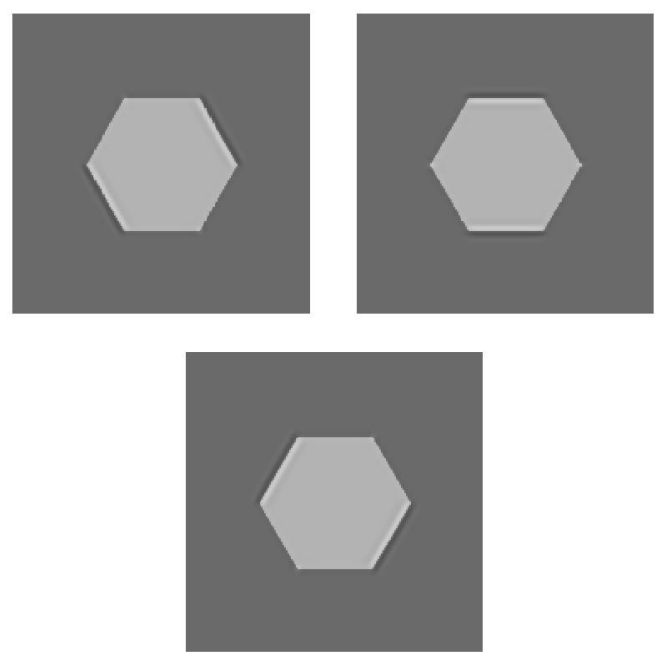

Fig. 7 - Steering the network with 2ODG connection.

\section{CONCLUSIONS}

The work presented in this paper reveals important theoretical aspects behind biologically inspired recurrent networks with lateral excitatoryinhibitory connectivity and addressed construction of filters arising from such networks. Spatially invariant inter-neuron coupling and the distribution of sensory units in the form of a uniform square grid yield the TBT connection matrix. The relationship between spectral properties of such matrices and their generating functions is utilized when constructing the network. For networks with the connection weights generated by the function whose absolute value is bounded by unity, the response can be determined without a large matrix inversion by the application of a single pass convolution. The filtering kernel is pre-computed based on a convergent matrix power series approximating the matrix inverse. Five different connection functions are analyzed and proper parameters determined when choosing weights of the network. Finally, it was shown that for the inter-neuron coupling in the form of a function expandable in a Fourier series in polar angle, the network impulse response is steerable.

It is clear that the presented work is limited to theoretical concepts related to construction of filters arising from the recurrent linear networks with spatially invariant inter-neuron coupling. Hopefully, the results provided herein could be used by other 
researchers to better understand and deploy techniques of signal processing based on such neural models. Utilization of filters described in this text for image processing and feature extraction applications when solving pattern recognition problems is currently being investigated by the authors and will be reported in a sequel paper.

\section{REFERENCES}

[1] H.K. Hartline, F. Ratliff, Inhibitory interaction of receptor units in the eye of Limulus, J. Gen. Physiol., (40) 3 (1957), pp. 357-376.

[2] H.K. Hartline, F. Ratliff, Spatial summation of inhibitory influences in the eye of Limulus, and the mutual interaction of receptor units, J. Gen. Physiol., (41) 5 (1958), pp. 1049-1066.

[3] B.S. Gutkin, C.E. Smith, Conditions for noise reduction and stable encoding of spatial structure by cortical neural networks, Biol. Cybern., (82) 6 (2000), pp. 469-475.

[4] G.G. Furman, Comparison of models for subtractive and shunting lateral-inhibition in receptor-neuron fields, Biol. Cybern., (2) 6 (1965), pp. 257-274.

[5] R.A. Messner, Smart Visual Sensors for RealTime Image Processing and Pattern Recognition Based Upon Human Visual System Characteristics, $\mathrm{PhD}$ Dissertation, Clarkson University, 1984.

[6] H.H. Szu, R.A. Messner, Adaptive invariant novelty filters, Proc. IEEE, (74) 3 (1986), pp. 518-519.

[7] S.E. Palmer, Vision Science Photons to Phenomenology, A Bradford Book, The MIT Press, Cambridge, Massachusetts, London, England, 1999.

[8] M.G. Luniewicz, R.A. Messner, Effects of lateral subtractive inhibition within the context of a polar-log spatial coordinate mapping, in: D.P. Casasent (Ed.), Proc. SPIE Intelligent Robots and Computer Vision VII, 1988, vol. 1002, pp. 58-65.

[9] Y. Yu, T. Yamauchi, Y. Choe, Explaining lowlevel brightness-contrast illusions using disinhibition, in A.J. Ijspeert, M. Murata, N. Wakamiya (Eds.), Biologically Inspired Approaches to Advanced Information Technology: First International Workshop, BioADIT 2004, Lausanne, Switzerland, 2004, Revised Selected Papers, Lecture Notes in Computer Science, 2004, vol. 3141, pp. 166175.

[10] Y. Yu, Y. Choe, Angular disinhibition effect in a modified Poggendorff illusion, in K.D. Forbus, D. Gentner, T. Regier (Eds.), Proceedings of the 26th Annual Conference of the Cognitive Science Society, Mahwah, NJ, USA, (2004), pp. 1500-1505.

[11] M. Wax, T. Kailath, Efficient inversion of Toeplitz-block Toeplitz matrix, IEEE Trans. Acoust, Speech Signal Process., (31) 5 (1983), pp. 1218-1221.

[12] N. Kalouptsidis, G. Carayannis, and D. Manolakis, On block matrices with elements of special structure, Proc. IEEE Int. Conf. on Acoustics, Speech and Signal Processing, ICASSP'82, Paris, France, (May 1982), vol. 7, pp. 1744-1747.

[13] A.E. Yagle, A fast algorithm for Toeplitzblock-Toeplitz linear systems, Proc. IEEE Int. Conf. on Acoustics, Speech, and Signal Processing, ICASSP'01, Salt Lake City, USA, (2001), vol. 3, pp. 1929-1932.

[14] S.J. Reeves, Fast algorithm for solving block banded Toeplitz systems with banded Toeplitz blocks, Proc. IEEE Int. Conf. on Acoustics, Speech, and Signal Processing, ICASSP'02, Orlando, USA, (2002), vol. 4, pp. 3325-3328.

[15] D.A. Bini, B. Meini, Solving block banded block Toeplitz systems with banded Toeplitz blocks, in F.T. Luk (Ed.), Proc. SPIE, Advanced Signal Processing Algorithms, Architectures, and Implementations IX, 1999, vol. 3807 pp. 300-311.

[16] U. Grenander, G. Szegö, Toeplitz Forms and Their Applications, 2nd ed., Chelsea Publishing, New York, 1984.

[17] S. Serra, Preconditioning strategies for asymptotically ill conditioned block Toeplitz systems, BIT Num. Math., (34) (1994), pp. 579594.

[18] P. Tilli, On the asymptotic spectrum of Hermitian block Toeplitz matrices with Toeplitz blocks, Math. Comp., (66) 219 (1997), pp. 1147-1159.

[19] W.T. Freeman, H.H. Adelson, The design and use of steerable filters, IEEE Trans. Pattern Anal. Mach. Intell., (13) 9 (1991), pp. 891-906.

[20] S. Barnet, Matrices: Methods and Applications, Oxford University Press, New York, 1990.

[21] R.A. Young, L.M. Lesperance, W.W. Meyer, The Gaussian derivative model for spatialtemporal vision: I. Cortical model, Spat. Vis., (14) 3,4 (2001), pp. 261-319.

[22] E.M. Stein, G. Weiss, Introduction to Fourier Analysis on Euclidean Spaces, Princeton University Press, Princeton, New Jersey, 1971.

[23] J.B. Price, M.H. Hayes, Steerable filter cascades, Proc. IEEE Intentional Conference on Image Processing ICIP'99, Kobe, Japan, (October 1999), vol. 2, pp. 880-884. 


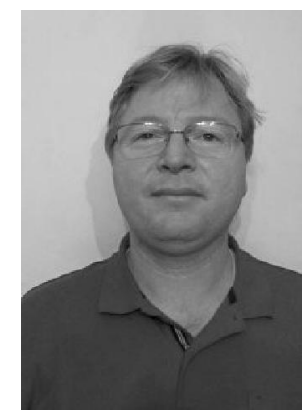

Dragan Vidacic received his Ph. D. and M. S. degrees in Electrical Engineering from the University of New Hampshire in 2009 and 2003 respectively as well as his B. S. also in Electrical Engineering from the University of Novi Sad in 2000. The main areas of his professional interest are Signal

Processing, Pattern Classification and Machine Vision. Presently, he is with Research and Development Engineering group at IDEXX Laboratories INC. where he is responsible for design of digital imaging technology for applications in life sciences.

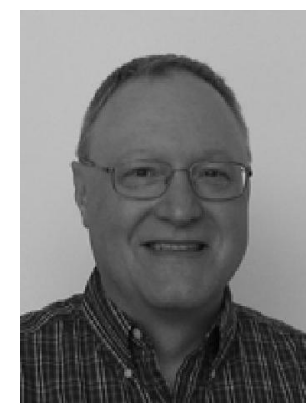

Dr Richard A. Messner received the B.S. and M.S. degrees from Clarkson College of Technology, Potsdam, NY in 1979 and 1981 respectively. From 1981 to 1982 Dr. Messner was a member of the technical staff at the MITRE Corporation in Bedford, MA. He recieved an ONR fellowship award and conducted his Ph.D. research in the Applied Optics Branch of the Optical Sciences Division at the Naval Research Laboratory in Washington, D.C. under the direction of Dr. Harold H. Szu. He was awarded the Ph.D. degree in 1985 from Clarkson University. The same year he joined the Deptartment of Electrical and Computer Engineering as an Assistant Professor and was promoted to Associate Professor in 1989. His resarch interests include hybrid optical/digital signal processing, real time digital image processing, and biologically inspired signal processing. Dr. Messner is a senior member of IEEE, SPIE, Eta Kappa Nu, and Life member of Sigma Xi. 